\title{
Prostacyclin and Interaction with Diacylglycerol Lipase and CDP Diacylglycerol; Possibility of De Novo Synthesisof Prostacyclin or Related Congeners by Novel Mechanisms
}

\author{
Manoj G Tyagi, Aniket Kumar, Shailendra K Vajpeyee* \\ Department of Pharmacology Jaipur National University Jaipur \\ Medical College, Surat, Gujarat
}

\begin{abstract}
Prostacyclin is a strong cardioprotective hormone released by the endothelium of the blood vessels. Prostacyclin is present in equilibrium with several vasoactive agents in cardiovascular system. In recent years, prostacyclin (PGI2) has also been shown to enhance differentiation and inhibit proliferation in vascular smooth muscle cells. In addition to these well-described homeostatic roles within the cardiovascular system, prostacyclin (PGI2) also plays an important role as an inflammatory mediator. In this review, the focus on the contribution of prostacyclin (PGI2) as both a patho-physiological mediator in three major inflammatorymediated disease processes, namely rheumatoid arthritis, where it promotes disease progression, along with pulmonary vascular disease and atherosclerosis, where it inhibits disease progression. On the other hand, $C D P-D A G$ synthases $(C D S)$ are enzymes that catalyze the conversion of phosphatidic acid (PA) to CDPdiacylglycerol (CDP-DAG). Both PA and CDP-DAG serve critical roles in cellular functions. This article reviews the possibility of interaction with CDP diacylglycerol and it appears that de novo synthesis of $P_{G} I_{2}$ or its congeners occurs in specialized cells under patho-physiological conditions.
\end{abstract}

Key words: Prostacyclin, diacylglycerol, enzyme, phosphatidic acid, vascular, inflammation

\section{Introduction}

Salvador Moncada showed in his exemplary work the importance of prostacyclin as an important autacoid and with vasodilatory properties and anti-platelet agent (1).Prostacyclin $\left(\mathrm{PGI}_{2}\right)$ is a potent vasorelaxant and an inhibitor of platelet aggregation. Prostacyclin is synthesized from free arachidonic acid, and is released from membrane phospholipids, which is converted into prostaglandin endoperoxide $\left(\mathrm{PGH}_{2}\right)$ by prostaglandin synthases (PGHS). $\mathrm{PGH}_{2}$ is subsequently metabolized to prostacyclin by the enzyme prostacyclin synthase. Prostaglandin $\mathrm{I}_{2}\left(\mathrm{PGI}_{2}\right)$ is the major metabolite of the cyclooxygenase pathway, and epoxyeicosatrienoic acids (EETs) are the products of the cytochrome P-450 epoxygenase pathway (2-4). PGI $_{2}$ relaxes vascular smooth muscle by increasing cAMP and opening ATP-sensitive potassium $\left(\mathrm{K}^{+}\right)$channels (5). EETs represent endothelium-derived hyperpolarizing factors.On the hand, the roles of CDS1 and CDS2 have primarily been studied in PI synthesis.Many of the cellular functions attributed to CDS enzymes are believed to result from their role in generating the precursor for phosphatidylinositol 4,5-bisphosphate $\left(\mathrm{PIP}_{2}\right)$, a potent signaling molecule (6). For example, photo-transduction signaling in vertebrate and invertebrate systems is believed to proceed, at least partly, via phosphoinositide signaling. Although $\mathrm{PLA}_{2}$ activation is the most direct route for arachidonic acid release in response to various stimuli, alternative pathways exist in some cells. Arachidonic acid may be released from diacylglycerol (DAG), generated via phospholipase C (PLC) activation, by the enzyme DAG lipase (7). Previous studies have shown that fibroblasts from cancer mass produce PGI2 but fibroblasts from adjacent normal tissues do not. However, how cancer associated fibroblasts (CAFs) are activated to produce $\mathrm{PGI}_{2}$ and if the activated $\mathrm{CAFs}$ can promote angiogenesis by generating VEGF itself are poorly understood.Recent experimental studies suggest the modulation of the gene expression in human fibroblasts under hypoxic condition using GeneChip analysis, and found that the expression of prostacyclin synthase (PGIS) was upregulated. PGIS, a membrane-bound heme protein with spectral characteristics of cytochrome p450 (CYP), is also an enzyme which catalyzes the conversion of prostaglandin $\mathrm{H}_{2}\left(\mathrm{PGH}_{2}\right)$ to form PGI2. PGIS is localized to the microsomal fractions of platelets, vascular endothelial cells, and vascular smooth muscle cells (8-9). Therefore this article evaluates the role of PGI2 in inflammation and interaction with CDP diacylglycerol and possibility of prostacyclin or related congeners synthesized by de-novo pathways.

PGI2 and signal transduction:PGI2 is unstable at physiological $\mathrm{pH}$ and, thus, has a very short half-life in vivo ( $<2 \mathrm{~min}$ ), rapidly transforming into the inactive hydration product 6-keto-prostaglandin F1 $\alpha$ or 6-oxo-PGF1 alpha (10-11). The actions of PGI2 are mediated through a seven-transmembrane-spanning G-protein coupled receptor (GPCR), referred to as the IP receptor (IUPHAR nomenclature) as shown in Fig.1. The IP receptor is a Class A rhodopsin-like GPCR that couples pre-dominately to the Gs subunit of the heterotrimeric G-protein and mediates intracellular signaling via adenylyl cyclase activation and cyclic AMP production (12). Des - 
aspartate-angiotensin 1 (DAA-I) peptide derivative has also been shown to release PGI2. The release of PGE2 and PGI2 via the angiotensin AT1 receptor and COX-1 is a novel specific action of DAA-I and is likely responsible for its beneficial effects seen in earlier studies. This specific action is definable as a biased agonism of the angiotensin AT1 receptor, which identifies DAA-I as a novel biased agonist and potential therapeutic that is able to produce specific prostaglandins at nanomolar concentrationsthus throwing light on possible novel mechanism for prostaglandins as well (13). Animal studies have also shown that PGI2 may also signal through alternateGq- and Gi-related pathways (14), as well as nuclear receptor-mediated pathways, such as the peroxisome proliferator activated receptor gamma (PPAR $\delta$ ) pathway (15).

PGI2 and postulated role in Inflammation:The seminal work by Vane (16) demonstrating the inhibition of prostaglandin biosynthesis as the mechanism of action for aspirin (acetylsalicylic acid) and other aspirin-like drugs first highlighted the importance of the prostaglandin family of molecules, and set the stage for the development of many pharmacologic agents, such as traditional, non-selective non-steroidal anti-inflammatory drugs (NSAIDs) and the newer selective COX-2 inhibitors. Further work conducted by Davies et al (17) pinpointed particular prostaglandins, principally prostaglandin E2 (PGE2) and prostacyclin (PGI2), in the mediation of vascular permeability associated with the hyperemia and edema seen with acute inflammation. Murata et al. (18) demonstrated the involvement of prostacyclin (PGI2)-mediated inflamma-tory swelling in vivo, using prostacyclin receptor deficient (IP-/-) mice. In these critical experiments, it was shown that mice lacking the prostacyclin receptor had a reduced inflammatory response, as measured by percent change in vascular permeability using a carrageenan-induced paw-edema model.

CDP diacylglycerol synthases: Only two CDP diacylglycerolsyntheases (CDS) isoforms in mammals have been identified and characterized. Both these isoforms are believed to be localized to the endoplasmic reticulum (ER). It was believed that CDS1 was present in mitochondria for synthesizing cardiolipin. CDS1 and CDS2 are expressed in a variety of tissues. In mice, CDS1 is found in adult brain, eye, smooth muscle, and testis (19). In the eyes, CDS1 is strongly expressed in the photoreceptor layer of adult retinas, which could suggest a role for CDS1 in phototransduction.CDS2 has a broad expression pattern and was found in virtually every tissue, however, some discrepancies exist in the tissue localization of CDS2 (20). The de novo synthesis of phosphoinositide (PI) involves only the endoplasmic reticulum and generates mainly saturated and monounsaturated acyl chains. The PI cycle is a cyclical pathway that involves the breakdown and regeneration of $\mathrm{PIP}_{2}$. The PI cycle involves both the endoplasmic reticulum and plasma membrane and results in the enrichment of 1-stearoyl-2-arachidonoyl species.Both pathways involve common features, one of which is the conversion of PA species to CDP-DAG by CDS enzymes. It appears likely that CDS2 would be involved in the PI cycle (19). The acyl chain selectivity of CDS is similar to that of DGKe, which was shown to be required for the arachidonoyl enrichment of PI species. CDS2 could play a similar yet important role in the enrichment of PI with an arachidonoyl chain. CDP-DAG produced by CDS2 can be used only for the synthesis of phospholipids (21-22).

Table 1:Effect of DAG lipase inhibitor RHC 80267 and IL-1 beta on 6-oxo-PGF1 alpha levels in human lymphocyte cells $(n=5)$

\begin{tabular}{|c|l|l|c|}
\hline Nos. & Pretreatment & Treatment & 6-oxo-PGF1alpha (pg/well) \\
\hline 1$)$ & Saline & Saline & $313 \pm 35.11$ \\
\hline 2$)$ & Saline & IL-1beta $(200 \mathrm{U} / \mathrm{ml})$ & $385 \pm 30.22$ \\
\hline 3$)$ & Saline & RHC80267 $(20 \mu \mathrm{M} / \mathrm{ml})$ & $165 \pm 23.72$ \\
\hline 4$)$ & IL1-beta & RHC 80267 & $225 \pm 27.97$ \\
\hline
\end{tabular}

Figure 1: Hypoxia and prostacyclin mediated signaling pathway

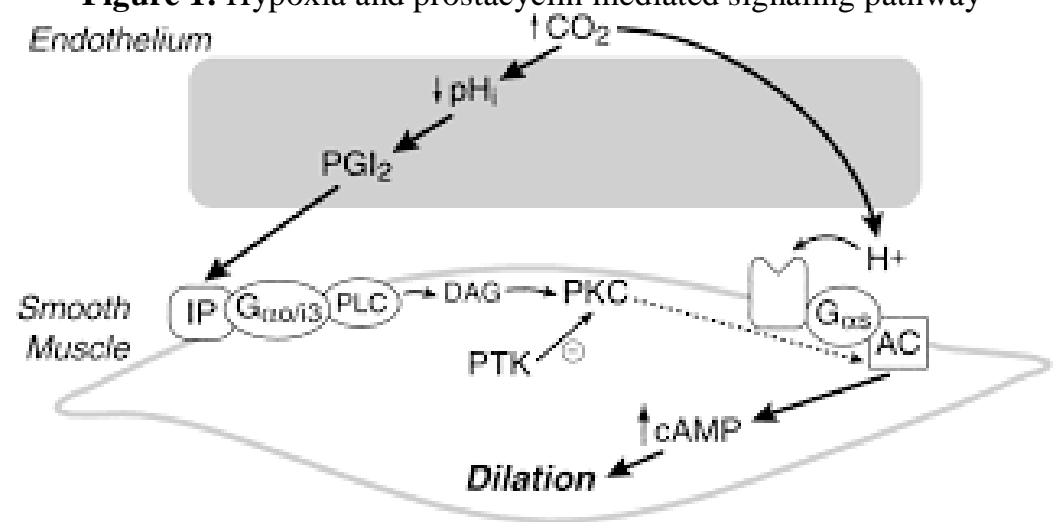




\section{Discussion}

Table 1 shows the effects of IL1 beta and DAG lipase inhibition by RHC 80267 on 6-oxo-PGF1 alpha levels as measured by radioimmunoassay. It appears that in lymphocyte cells the RHC 80267 either directly inhibits the the prostacyclin synthesis or its congeners or otherwise the accumulation of diacylglycerol causes the inhibition of prostacyclin or its putative congeners via some unspecified mechanisms. On the other hand interleukin 1 beta was able to increase the levels of PGI2 and this was attenuated by the known DAG lipase inhibitor RHC 80267. The work done by D'Souza et al is suggestive of the importance of CDS enzyme and apparently there is more research work required in this area to confirm the exact role played by diacylglycerol and related pathways and the synthesis of prostacyclin like congeners (23-24). Thus it is still an area where more research work needs to be carried out and the exact phenomenon elucidated.

\section{Conclusions}

Prostacyclin is an important prostaglandin which has important vasodilatoryand anti-platelet activity and roles beyond the cardiovascular system. Our existing knowledge of $\mathrm{PGI}_{2}$, as both a physiologicalpathophysiological mediator and therapeutic agent, in a host of inflammatory-related diseases, is growing rapidly. As demonstrated by several studies, PGI2 has been shown to play protective roles in atherogenesis relating to $\mathrm{CAD}, \mathrm{MI}$, stroke, and other cardiovascular abnormalities. It has also been shown to be involved in certain fibro-proliferative and pulmonary vascular diseases, such as idiopathic pulmonary fibrosis and pulmonary hypertension, where it serves as both a protective factor and first-line pharmacotherapy. It appears from some recent studies that prostacyclin or its congeners may be synthesized by novel de-novo pathways. Further study of this important prostaglandin, in both the realms of basic science and clinical medicine, is required and will undoubtedly lead to new insights into inflammatory disorders and pharmacological treatments.

\section{References}

[1] Moncada S, Gryglewski R, Bunting S and Vane JR: An enzyme isolated from arteries transforms prostaglandin endoperoxides to an unstable substance that inhibits platelet aggregation. Nature. 263:663-665. 1976.

[2] Rosolowsky M and Campbell WB. Role of PGI2 and epoxyeicosatrienoic acids in relaxation of bovine coronary arteries to arachidonic acid. Am J Physiol Heart CircPhysiol 264: H327-H335, 1993

[3] Ullrich V, Castle L and Weber P: Spectral evidence for the cytochrome P450 nature of prostacyclin synthetase. BiochemPharmacol. 30:2033-2036. 1981.

[4] M G Tyagi, H Kan, Y Ruan, KU Malik. Studies on the Characterization of the Subtype(S) of Muscarinic Receptor Involved in Prostacyclin Synthesis in Rabbit Cardiomyocytes. Journal of Receptor and Signal Transduction Research, 1996; 16(5-6): 273-96

[5] Li PL, Zou AP, and Campbell WB. Regulation of potassium channels in coronary arterial smooth muscle by endothelium-derived vasodilators. Hypertension 29: 262-267, 1997.

[6] Lohn M, Dubrovska G, Lauterbach B, Luft FC, Gollasch M, and Sharma AM. Periadventitial fat releases a vascular relaxing factor. FASEB J 16: 1057-1063, 2002.

[7] Lykidis, A., Jackson, P. D., Rock, C. O., and Jackowski, S. (1997) The role of CDP-diacylglycerolsynthetase and phosphatidylinositol synthase activity levels in the regulation of cellular phosphatidylinositol content. J. Biol. Chem. 272, 33402-33409.

[8] Pratt PF, Rosolowsky M, and Campbell WB. Mediators of arachidonic acid-induced relaxation of bovine coronary artery. Hypertension 28: 76-82, 1996.

[9] Cutler NS, Graves-Deal R, LaFleur BJ, Gao Z, Boman BM, Whitehead RH, Terry E, Morrow JD and Coffey RJ: Stromal production of prostacyclin confers an antiapoptotic effect to colonic epithelial cells. Cancer Res. 63:1748-1751. 2003

[10] Lewis, P. J., and Dollery, C. T. (1983). Clinical pharmacology and potential of prostacyclin. Br. Med. Bull. 39, 281-284.

[11] Smyth, E. M., and FitzGerald, G. A. (2002). Human prostacyclin receptor. Vitam. Horm. 65, 149-165.

[12] Boie, Y., Rushmore, T. H., Darmon- Goodwin, A., Grygorczyk, R., Slipetz, D. M., Metters, K. M., and Abramovitz, M. (1994). Cloning and expression of a cDNA for the human prostanoid IP receptor. J. Biol. Chem. 269, 12173-12178.

[13] Wen Q, Lee KO, SimSZ, Xu XG, SimMK.Des-aspartate-angiotensin I causes specific release of PGE2 and PGI2 in HUVEC via the angiotensin AT1 receptor and biased agonism.Eur J Pharmacol. 2015;768:173-81

[14] Lawler, O. A., Miggin, S. M., and Kinsella, B. T. (2001). Protein kinase A-mediated phosphorylation of serine 357 of the mouse prostacyclin receptor regulates its coupling to G(s)-, to G(i)-, and to G(q)-coupled effector signaling. J. Biol. Chem. 276, 3359633607 .

[15] Lim, H., and Dey, S. K. (2002). A novel pathway of prostacyclin signaling- hanging out with nuclear receptors. Endocrinology 143, 3207-3210.

[16] Vane, J. R. (1971). Inhibition of prostaglandin synthesis as a mechanism of action for aspirin-like drugs. Nat. New Biol. 231, 232235

[17] Davies P., Bailey P. J., Goldenberg M. M., Ford-Hutchinson A. W. (1984). The role of arachidonic acid oxygenation products in pain and inflammation. Annu. Rev. Immunol. 2, 335-35710

[18] Murata, T., Ushikubi, F., Matsuoka, T., Hirata, M., Yamasaki, A., Sugimoto, Y., Ichikawa, A., Aze, Y., Tanaka, T., Yoshida, N., Ueno, A., Oh-Ishi, S., and Narumiya, S. (1997). Altered pain per-ception and inflammatory response in mice lacking prostacyclin receptor. Nature 388, 678-682.

[19] Saito, S., Goto, K., Tonosaki, A., and Kondo, H. (1997) Gene cloning and characterization of CDP-diacylglycerol synthase from rat brain. J. Biol. Chem. 272, 9503-9509.

[20] Volta, M., Bulfone, A., Gattuso, C., Rossi, E., Mariani, M.,Consalez, G. G., Zuffardi, O., Ballabio, A., Banfi, S., and Franco, B. (1999) Identification and characterization of CDS2, a mammalian homolog of the Drosophila CDP-diacylglycerol synthase gene.Genomics 55, 68-77.

[21] Inglis-Broadgate, S. L., Ocaka, L., Banerjee, R., Gaasenbeek, M., Chapple, J. P., Cheetham, M. E., Clark, B. J., Hunt, D. M., and Halford, S. (2005) Isolation and characterization of murine Cds (CDPdiacylglycerol synthase) 1 and 2. Gene 356, $19-31$. 
[22] Mok, A. Y., McDougall, G. E., and McMurray, W. C. (1993) Comparative studies of CDP-diacylglycerol synthase in rat liver mitochondria and microsomes. Biochem. Cell Biol. 71, 183-189.

[23] Tyagi MG.An evaluation of the role of phospholipase Denzymes in blood cells and possible implications for health homeostasis. EJPMR, 2016,3(11), 279-283

[24] KD'Souza, YJu Kim,TBalla,and R.M. EpandDistinct Properties of the Two Isoforms of CDP-Diacylglycerol Synthase.Biochemistry. 2014 Dec 2; 53(47): 7358-7367. 